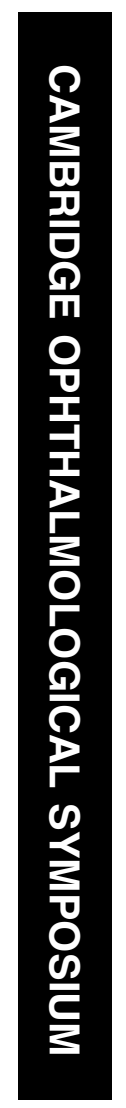

\section{Development of the human choriocapillaris}

GA Lutty', T Hasegawa', T Baba', R Grebe', I Bhutto' ${ }^{1}$ and DS McLeod ${ }^{1}$

Keywords: choriocapillaris; fenestrations; foetal; haemo-vasculogenesis; pericytes; ultrastructure

Introduction

Human choroid is a thin, highly vascularized and pigmented tissue positioned under the sensory retina that forms the posterior portion of the uveal tract (the iris, ciliary body, and choroid). The inner boundary of the choroid is Bruch's membrane (BrM) on which the retinal pigment epithelium (RPE) monolayer is present. This vasculature has three layers: the anterior choriocapillaris (CC) with broad, flat lumens (20-50 $\mu \mathrm{m}$ diameter) arranged in a honeycomblike lobular pattern especially in the posterior pole; Sattler's layer of intermediate vessels in the middle; and the outermost Haller's layer with large vessels. ${ }^{1}$ The choroidal vasculature is responsible for maintaining the metabolic demands of the RPE cells and photoreceptors; therefore, abnormalities in this vasculature result in many kinds of congenital and adult diseases such as choroidal coloboma and agerelated macular degeneration. ${ }^{2-4}$

The nutrients for photoreceptors are transported through the fenestrations of the CC. Most studies suggest that these unique structures exist only on the retinal side of CC towards the RPE, however, we have observed them on the scleral side of lumens as well. ${ }^{5}$ The $\mathrm{CC}$ is sided also in disposition of VEGF receptors as they are on the retinal side presumably because VEGF is secreted from the basal side of the RPE, which is thought to be necessary in maintaining the $\mathrm{CC}$ fenestrations. ${ }^{6}$ The intent of this review is to summarize our studies on the development and maturation of the human CC.

The initial CC develops by haemo-vasculogenesis (6-8 weeks gestation)

Vascular development occurs predominantly by three processes: vasculogenesis, coalescence 
and assembly of vascular precursors or angioblasts; angiogenesis, migration and proliferation of endothelial cells (ECs) from a pre-existing blood vessel; and haemovasculogenesis, the differentiation of blood cells and blood vessel cells from a common precursor, the haemangioblast. We have recently shown that the initial human CC develops by haemo-vasculogenesis between 6 and 8 weeks gestation (WG). ${ }^{7}$ At $6-7$ WG, erythroblasts (nucleated erythrocytes expressing $\varepsilon$ haemoglobin $\left(\mathrm{Hb}-\varepsilon^{-}\right)$) were observed within islands of progenitors (blood island-like formations) in the CC layer and scattered within the forming choroidal stroma (Figure 1). Often the same cells that expressed $\mathrm{Hb}-\varepsilon$ co-expressed EC (CD31, CD34, CD39), haematopoietic (CD34), and angioblast (CD39, VEGFR-2) markers (Figure 2) suggesting that these cells were haemangioblasts or progenitors derived from haemangioblasts and that haemo-vasculogenesis is the process by which the initial CC develops. ${ }^{7}$ We observed only a single layer of vasculature in choroid at this time, the forming CC (Figure 3). By 8.5 WG, there are very few erythroblasts associated with $\mathrm{CC}$ and vascular lumens become apparent. Development of the CC from islands of progenitors explains how this vasculature forms without a source of blood, i.e. no large vessels were present. $^{7}$ Haemovasculogenesis has been observed in several organ systems in embryonic mouse. ${ }^{8}$

\section{9-12 WG}

At 11-12 WG, development of deeper choroidal vessels was observed and this was more advanced in the posterior pole

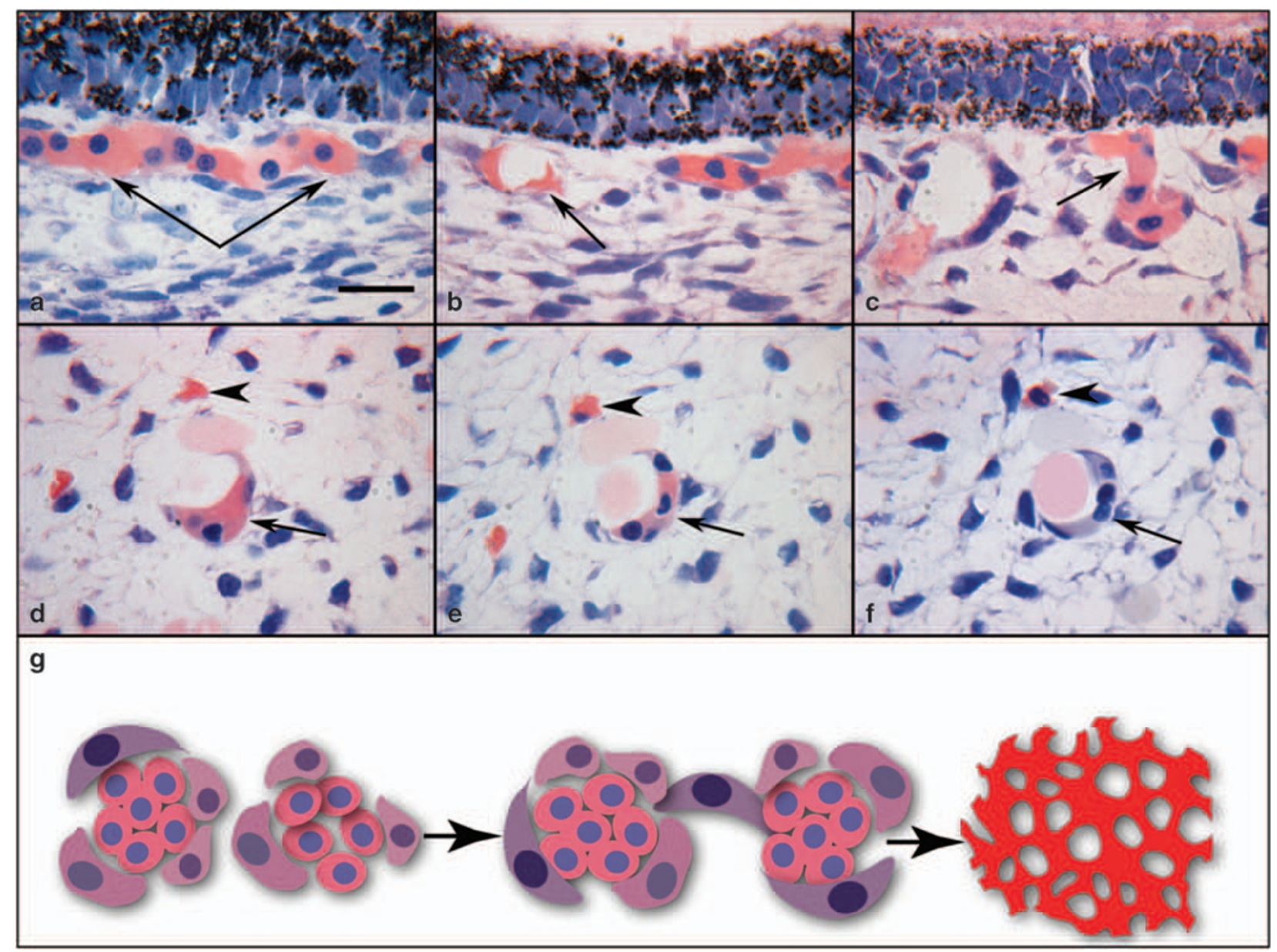

Figure 1 Haemo-vasculogenesis in cross-sections of 6.5 WG foetal choroid (a-f) and a schematic representation of this process (g). In the choriocapillaris (CC) layer, erythroblasts (bright pink cytoplasm) can form a solid cord-like structures (double arrow) without a lumen (a). Erythroblasts, haematopoetic and vascular cells develop in situ with erythroblasts sometimes forming lumen (arrow)(d). Eventually, the outer cells become primarily ECs and the inner cells become primarily erythroblasts (f). Free erythroblasts (arrowheads in $\mathrm{d}-\mathrm{f}$ ) are present in stroma of choroid. (g) A schematic of haemo-vasculogenesis in CC where islands of progenitors are then united by other progenitors eventually yielding a chicken-wire pattern that will become lobular in the adult CC. (Scale bar $=10 \mu \mathrm{m}$; $(\mathrm{a}-\mathrm{f})$ Giemsa stained JB4 sections). 

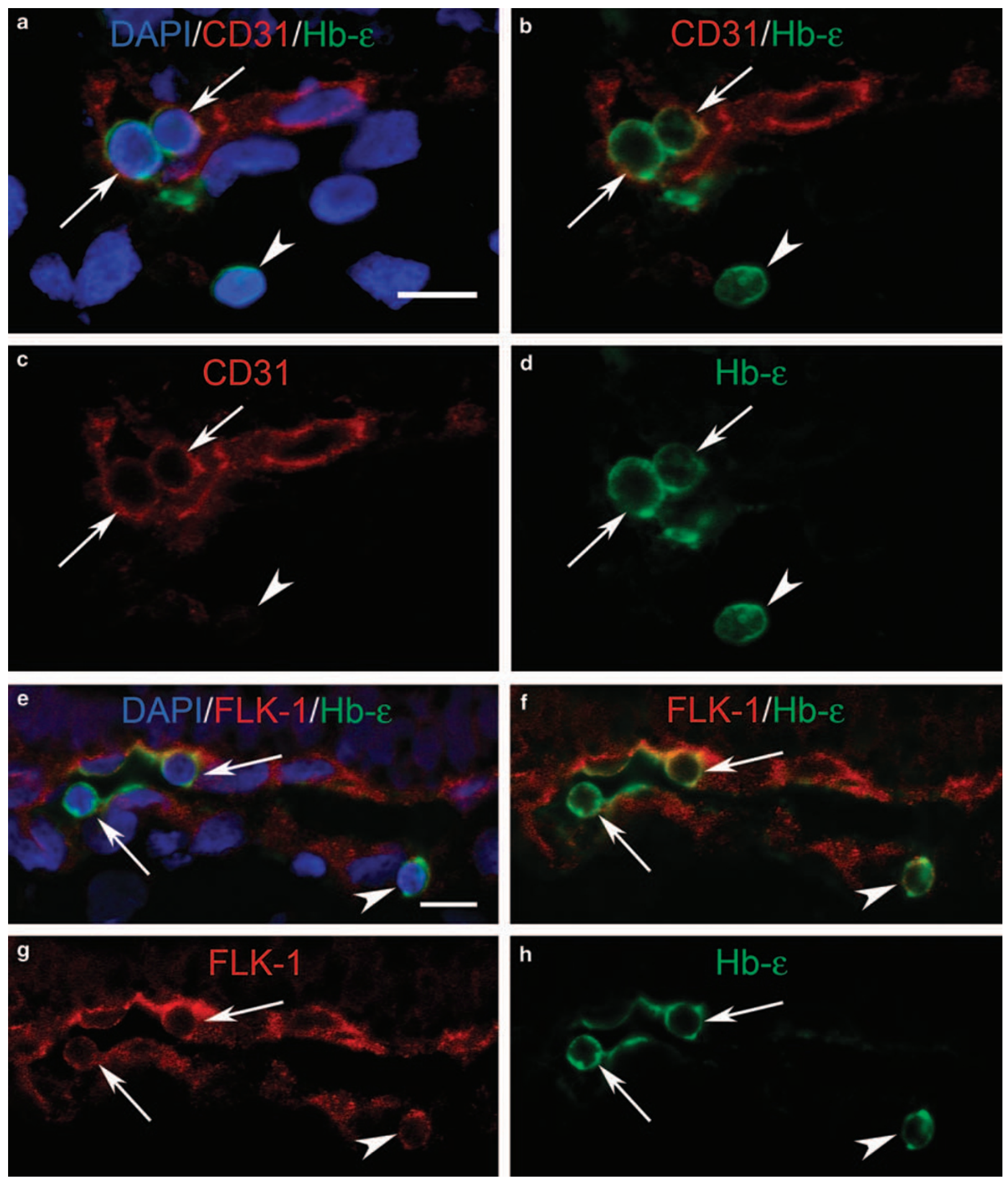

Figure 2 Co-localization of erythroid ( $\varepsilon$ haemoglobin, $\mathrm{Hb}-\varepsilon$ ) and endothelial markers (CD31 and VEGFR-2 or FLK-1) in developing choriocapillaris (CC). (a-d) CD31 (red) and Hb- $\varepsilon$ (green) are co-localized in cells of the developing CC (arrows) and single cells within the choroidal stroma (arrowhead). (e-h) FLK-1 and Hb- $\varepsilon$ co-expression in cells lining a developing lumen (arrows) and in cells located outside of the structure (arrowhead). (Scale bars $=10 \mu \mathrm{m}$; counterstained with DAPI, blue).

than in equatorial choroid (Figure 3). These vessels were positive for the EC markers including CD31, CD34, and CD39. Double labelling with CD34 and Ki67 showed that some ECs were proliferating and budding from the scleral side of CC, suggesting that intermediate vessels form by angiogenesis. ${ }^{7}$ Flat CD39-labelled preparations of choroid 


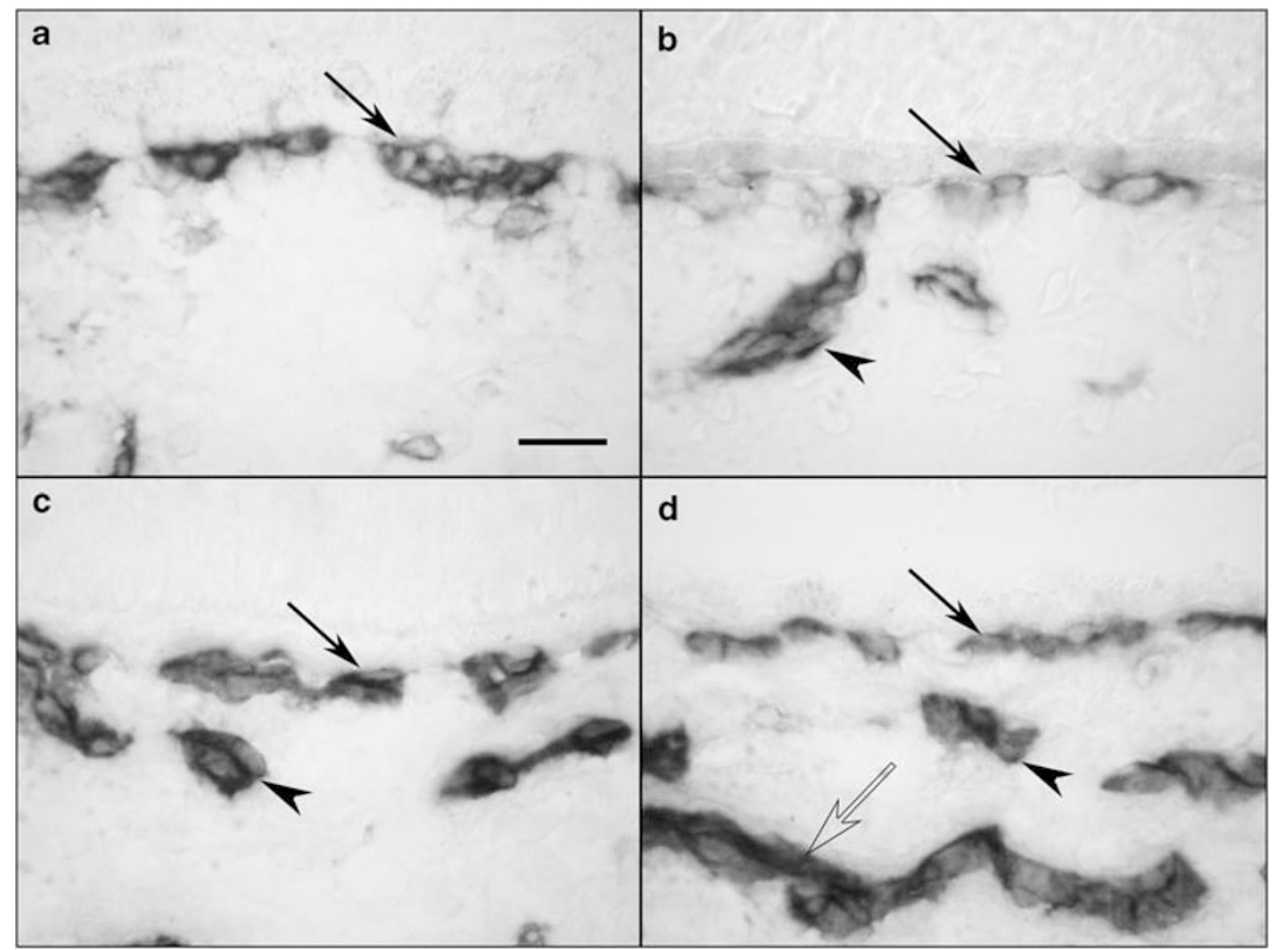

Figure 3 CD31 immunolabelling of choroidal sections from foetal eyes at 6 WG (a), 12 WG (b), 16 WG (c), and 20 WG (d). At 6 WG (a), only a highly cellular rudimentary choriocapillaris (CC) with poorly defined lumen (arrow) present. At 12 WG (b), vessels are diving from the CC into the deeper choroid (arrowhead). By 16 WG (c), well-defined CC lumens are present (arrow) and a network of medium-size deeper blood vessels has formed (arrowhead). At 22 WG (d), the CC (arrow), medium-size vessels of the Sattler's layer (arrowhead), and the larger outer blood vessels (open arrow) are all present. (Scale bar $=30 \mu \mathrm{m}$ ).

at 12 WG (Figure $4 \mathrm{~b}$ ) demonstrated a chicken-wire-like pattern of blood vessels with free CD39-positive cells between the vascular segments, ${ }^{7}$ suggesting that angioblasts were present in choroid. ${ }^{9}$

All vessels were negative for PV-1, plasmalemmal vesicle-associated protein, which is an integral membrane glycoprotein in the diaphragms of fenestrations. ${ }^{10,11}$ TEM demonstrated occasional fenestrations associated with the filipodial-like structures both in and around the lumen, but these fenestrations may not to be functional because of their unusual position. Basal lamina was not observed around these developing vessels.

Ultrastructurally, the CC was composed of aggregates of progenitors with only slit-like lumens (Figure 4c). ${ }^{12}$ Although some cells have assumed an adventitial position relative to the cells lining the primitive lumens, there was no difference in appearance of chromatin or organelles between the cells in peripheral CC (Figure 4c). All of the progenitors were plump in stature and there were some tight junctions present between the cells that bordered the lumens. More definitive pericyte-like cells were found adjacent to more developed vessels in central choroid (area from disc to equator). ${ }^{5}$ In the more mature central blood vessels, the pericytes had a nucleus that appeared more differentiated with distinct organelles in cytoplasm, whereas the ECs still had condensed chromatin and dense cytoplasm. The slit-like lumens were often filled with complex membranous infoldings that resembled filopodial processes from the luminal cells (Figure 4c). In some lumens at the equator that were more open, the filopodia appeared to touch erythrocytes in the lumen and the plasma membranes of the two cells could not be discerned. ${ }^{5}$

\section{4-16 WG}

By 14 WG in peripheral CC, cells in the ablumenal position of a pericyte formed peg-in-socket-like contacts with ECs lining the lumen, a characteristic of normal adult microvasculature. ${ }^{5}$ We used antibodies for two pericyte markers to evaluate the maturation of these abluminal cells: alpha smooth muscle actin (aSMA), present in mature pericytes, and NG-2, a glycosaminoglycan present on the surface of pericytes. There was limited aSMA immunoreactivity at 14 WG, whereas NG-2 immunoreactivity was very prominent.

At $16 \mathrm{WG}$, some areas of CC were weakly positive with PV-1 antibody suggesting the presence of some fenestrations. TEM confirmed that there were a few fenestrations in the $\mathrm{CC}$ at this age but they were scarce 


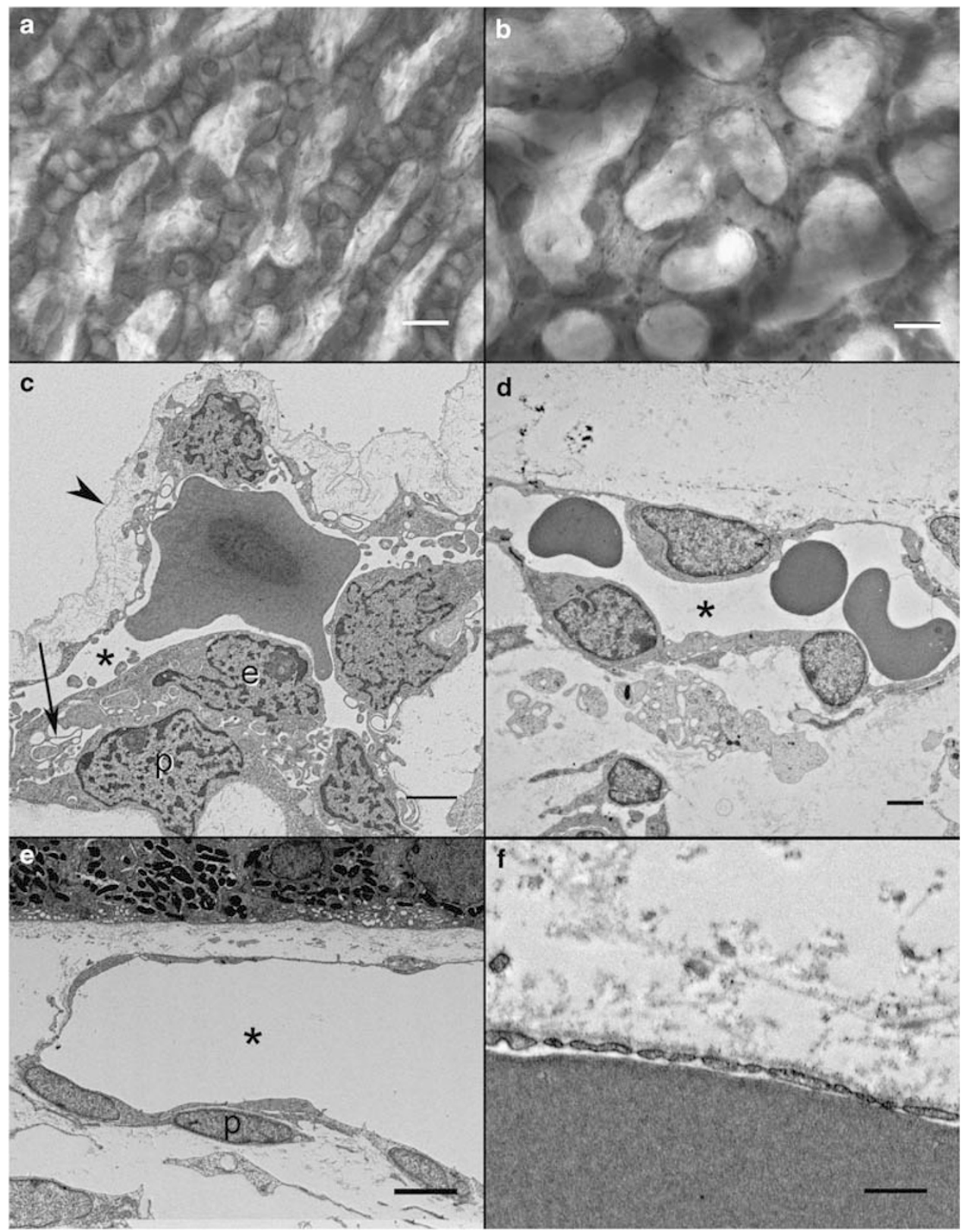

Figure 4 CD39 immunolabelled flat choroids showing the CC pattern $(a, b)$ and TEM images showing the structure of developing CC (c-f). At 9 WG (a), CD39-positive cells are organized into highly cellular solid cord-like structures without apparent lumen. By 12 WG (b), the capillaries have thinned, lumen have formed, and cellularity has decreased markedly. (c) In ultrathin sections from 11 WG choroid, plump EC nuclei (e) with condensed chromatin line a slit-like lumen (asterisk). Cytoplasmic extensions (arrow) projected into the lumenal space and in some cases made intimate contact with erythroblasts. Perivascular cells, putative pericytes (p), had ultrastructural features identical to the immature ECs. (d) In sections from a 16 WG choroid, lumenal spaces were more apparent (asterisk), the EC nuclei had finer chromatin, were reduced in volume, and had decreased cytoplasmic projections. (e) By 22 WG, lumens were broad and flat, ECs had thinned and definitive pericytes (p) were present on the outer surface of the capillaries. At this stage of development, fenestrations were present along inner aspect of the CC. (f) Fenestrations on the retinal side of a CC lumen at 22 WG. (Scale bars $=20 \mu \mathrm{m}(\mathrm{a}, \mathrm{b}) ; 2 \mu \mathrm{m}$ (c, d); $4 \mu \mathrm{m}$ (e); and $50 \mathrm{~nm}$ (f)).

and not continuous. The number of fenestrations was greatest in the posterior pole where the CC was most mature morphologically. The number of filopodia in these broader lumens appeared greatly reduced compared with 11 WG (Figure 4d). EC nuclei were more oval and uniform in shape with less dense chromatin. The rough endoplasmic reticulum appeared less dispersed. EC junctions were slightly more pronounced and BrM appeared more organized. Basal lamina was present but was more apparent on the retinal side of the capillary. 


\section{1-22 WG}

At 21 WG, three layers of blood vessels were apparent within the posterior pole region as demonstrated with EC markers. This is the first time point at which there is evidence of photoreceptor maturation. Short rudimentary inner segments were present at the outer most portion of the neuroblastic layer. PV-1 immunoreactivity was present in most of the CC but again it was more intense in the posterior pole than in periphery. However, in the adult human eye used as a positive control, the PV-1 was uniformly intense and more apparent on the retinal side of the CC lumens, whereas the other EC markers were uniform around the CC. ${ }^{5}$

TEM at 22 WG showed that the CC was now thin-walled, flat blood vessels with open lumens and contiguous areas of fenestrations (Figures $4 \mathrm{e}-\mathrm{f}$ ). Wellformed tight junctions with defined zonulae were present. BrM was also more developed with collagen and elastin dispersed under the RPE basement membrane.

aSMA increased continuously with age until 22 WG when $\mathrm{SMA}^{+}$cells were present throughout the CC and also around intermediate and large choroidal blood vessels. NG-2 was most prominent at 22 WG when pericytes were apparent by TEM at this age (Figure 4e). Their nuclei were oval and had homogenous chromatin, whereas their cytoplasmic processes were thin and alligned with the EC processes.

\section{Discussion}

Ida Mann elegantly documented the development of human CC by light microscopy. ${ }^{13}$ Using modern techniques, our studies show that the development of the human choroidal vasculature involves several processes. The initial human CC forms by haemo-vasculogenesis: the same cells were expressing $\mathrm{Hb}-\varepsilon$ as well as CD31, CD34, VEGFR-2, or vWf, further suggesting the same precursors were capable of erythropoiesis, haematopoiesis, and vasculogenesis, the definition of haemo-vasculogenesis, as occurs in blood islands. ${ }^{7}$ In the foetal period, haemo-vasculogenesis was complete and new blood vessels appeared to form by angiogenesis since ECs were proliferating (Table 1). ${ }^{7}$ Angiogenesis seems to contribute to development of the intermediate blood vessels and anastomosis between the capillaries and larger vessels as observed by Drake and associates in mouse. ${ }^{14}$ In the ages included in our studies, 6-22 WG, the CC never reached the lobular pattern in the posterior pole or ladder pattern in periphery or vascular density of the adult, ${ }^{15}$ suggesting that significant expansion and remodelling of the system will occur after 22 WG. This may occur by the process of vasculogenesis because free
$\mathrm{CD} 9^{+}$angioblasts were still present in between formed segments of CC. Alternatively, the additional segments may form by angiogenesis because proliferation is still occurring in CC at 22 WG.

Transport to and from CC occurs at least in part through fenestrations. Even though contiguous fenestrations are present at $22 \mathrm{WG}$, the relationship and interactions between CC and RPE are incomplete in that $\mathrm{BrM}$ is very immature. The highly metabolically active photoreceptors are also dependant on the CC for removal of the end products after the photoreceptor outer segment shedding and RPE digestion. Obviously, shedding is not occurring at 22 WG because only inner segments are present, but certainly their metabolism has increased as inner segments are populated by the mitochondria.

One of the final events in maturation of a blood vessel is the investment by adventitial cells: pericytes around capillaries and venules and SM cells (SMC) in the walls of arterioles and arteries. Again, little is known regarding the origins, differentiation, and appearance of contractile cells associated with the choroidal vasculature during embryonic and foetal development. TEM at 11 WG demonstrated perivascular and luminal cells had similar ultrastructural characteristics, suggesting that pericytes and ECs may have a common origin. Although NG-2 was present in these vascular structures at 11 WG, aSMA, the predominant actin isoform found in mature SMC and pericytes, ${ }^{16}$ was not present until 22 WG (Table 1). Our in vitro studies of retinal angioblasts show that the same progenitor may differentiate into either EC or pericyte depending on conditions. ${ }^{17}$ TEM at 22 WG demonstrated that apparent mature pericytes were present predominantly on the scleral side (Figure 4e).

Lumen formation is a key event in blood vessel maturation. As just mentioned, the initial lumenal spaces were slit-like and the cells lining them appeared as rotund mesenchymal precursors, as observed by Sellheyer as early as $6.5 \mathrm{WG}^{12}$ when haemovasculogenesis is occurring. ${ }^{7}$ Even at this stage the cells made recognizable tight junctions that are necessary for a mature vasculature, suggesting that these lumenal cells were committed to being EC lineage. A striking characteristic of the immature lumens at 11 WG was the extensive processes that were present within the luminal space. These processes resembled filopodia, slender cytoplasmic projections that are associated with endothelial migration, budding, and formation of new blood vessels. Roy and associates observed cytoplasmic extensions from developing ECs in chick brain and the number of cytoplasmic extensions decreased as the lumens became broader, ${ }^{18}$ as we observed in CC. ECs and angioblasts use these processes to touch and interlock with each other. ${ }^{19}$ It is not until 22 WG that the 
Table 1 Time line of choriocapillaris development

\begin{tabular}{|c|c|c|c|c|c|c|c|c|c|c|c|c|c|}
\hline WG & 7 & 8 & 9 & 10 & 11 & 12 & 14 & 16 & 17 & 20 & 21 & 22 & Adult \\
\hline \multicolumn{14}{|l|}{ Endothelial cells } \\
\hline $\mathrm{CD} 1^{\mathrm{a}}$ & + & & + & & & + & & & + & & + & & + \\
\hline $\mathrm{CD} 34^{\mathrm{b}}$ & + & & + & & & + & & & + & & + & & + \\
\hline PAL-E & + & & + & & & + & & & + & & + & & + \\
\hline \multicolumn{14}{|l|}{ Fenestrations } \\
\hline $\mathrm{PV}-1^{\mathrm{a}}$ & - & & - & & & - & & & Week & & + & & + \\
\hline TEM $^{\mathrm{a}}$ & & & & & None & & & A few & & & & Many & Many \\
\hline \multicolumn{14}{|l|}{ Pericytes } \\
\hline $\mathrm{SMA}^{\mathrm{b}}$ & - & & & & & + & & & & & & + & + \\
\hline $\mathrm{NG}-2^{\mathrm{b}}$ & Week & & & & & + & & & & & & + & + \\
\hline TEM $^{\mathrm{a}}$ & & & & & $\mathrm{pp}^{+} /$peri $^{-}$ & & & & & & & Definitive & Many \\
\hline Haemo-Vas $\left(\mathrm{Hb}-\varepsilon^{+}\right)^{\mathrm{b}}$ & & + & \pm & & & - & - & & - & & - & & - \\
\hline Angiogenesis $\left(\mathrm{Ki}^{6} 7^{+}\right)^{\mathrm{b}}$ & & - & \pm & & & + & & & + & & + & & - \\
\hline
\end{tabular}

${ }^{\mathrm{a} D a t a}$ from reference \#5.

bData from reference \#7.

Haemo-vas, haemo-vasculogenesis; TEM, transmission electron microscopy.

lumens are broad and flat as observed in the mature CC. At this time point, the ECs are fusiform, the wall of the blood vessel is thin, and pericytes have assumed a flatter profile and ensheath the blood vessels with their processes.

The CC is one of the few fenestrated capillary beds in the body. Fenestrations are unique pore-like structures that have a diaphragm, which is recognized by the PV-1 antibody. They allow passive transit of some fluids and macromolecules, which is critical in their providing outer retina, including RPE, with nutrients, ions, and oxygen as well as transport of the waste from the RPE. It was not until 22 WG that contiguous fenestrations were observed in some areas and PV-1 immunoreactivity was greatly increased in CC.

The development of the CC by haemo-vasculogenesis probably bestows certain unique developmental characteristics. Morphologically, it appears that the progenitors differentiate into either ECs or pericytes, although this needs to be further demonstrated with immunohistochemistry using cell markers. Formation of capillaries from islands of progenitors may contribute to formation of the lobular pattern of CC in that the first blood vessels are islands, which eventually connect to each other without any contribution from blood flow because intermediate and large blood vessels are not yet present (Figures 3 and 4), similar to the sequence of events in kidney development. ${ }^{20}$ The final mature CC is very similar to the capillaries of kidney glomeruli: large, flat, fenestrated capillaries that are lobular in pattern. ${ }^{20}$ Fenestrations form late in maturation (21-22 WG), which nicely anticipates the differentiation of photoreceptors that Hendrickson and co-workers have shown begins around 24-26 WG when inner segments form. ${ }^{21}$ After this time, the CC will be sided, fenestrated mostly on the RPE side, which is critical for its adult function in supporting the viability of photoreceptors and RPE cells.

\section{Conflict of interest}

The authors declare no conflict of interest.

\section{Acknowledgements}

This work was supported in part by NIH-EY-016151 (GAL), EY-01765 (Wilmer), the Altsheler-Durell Foundation, and a gift from the Himmelfarb Family Foundation in the name of Morton F Goldberg. Takuya Hasegawa and Takayuki Baba were Bausch and Lomb Japan Vitreoretinal Research Fellows, and Takayuki Baba was also a Uehara Memorial Foundation Research Fellow.

\section{References}

1 Bhutto IA, Lutty GA. The vasculature of choroid. In: Schepro D, D'Amore PA (eds). Encyclopedia of Microvasculatures. Elsevier: San Diego, 2004.

2 Daufenbach DR, Ruttum MS, Pulido JS, Keech RV. Chorioretinal colobomas in a pediatric population. Ophthalmology 1998; 105(8): 1455-1458.

3 Lutty G, Grunwald J, Majji AB, Uyama M, Yoneya S. Changes in choriocapillaris and retinal pigment epithelium in age-related macular degeneration. Mol Vis 1999; 5: 35.

4 McLeod DS, Grebe R, Bhutto I, Merges C, Baba T, Lutty GA. Relationship between RPE and choriocapillaris in agerelated macular degeneration. Invest Ophthalmol Vis Sci 2009; 50(10): 4982-4991. 
5 Baba T, Grebe R, Hasegawa T, Bhutto I, Merges C, McLeod DS et al Maturation of the fetal human choriocapillaris. Invest Ophthalmol Vis Sci 2009; 50(7): 3503-3511.

6 Blaauwgeers HG, Holtkamp GM, Rutten H, Witmer AN, Koolwijk P, Partanen TA et al Polarized vascular endothelial growth factor secretion by human retinal pigment epithelium and localization of vascular endothelial growth factor receptors on the inner choriocapillaris. Am J Pathol 1999; 155: 421-428.

7 Hasegawa T, McLeod DS, Bhutto IA, Prow T, Merges CA, Grebe $\mathrm{R}$ et al The embryonic human choriocapillaris develops by hemo-vasculogenesis. Dev Dyn 2007; 236 2089-2100.

8 Sequeira Lopez ML, Chernavvsky DR, Nomasa T, Wall L, Yanagisawa M, Gomez RA. The embryo makes red blood cell progenitors in every tissue simultaneously with blood vessel morphogenesis. Am J Physiol Regul Integr Comp Physiol 2003; 284(4): R1126-R1137.

9 McLeod DS, Hasegawa T, Prow T, Merges C, Lutty G. The initial fetal human retinal vasculature develops by vasculogenesis. Dev Dyn 2006; 235: 3336-3347.

10 Stan RV, Ghitescu L, Jacobson BS, Palade GE. Isolation, cloning, and localization of rat PV-1, a novel endothelial caveolar protein. J Cell Biol 1999; 145(6): 1189-1198.

11 Stan RV, Kubitza M, Palade GE. PV-1 is a component of the fenestral and stomatal diaphragms in fenestrated endothelia. Proc Natl Acad Sci USA 1999; 96(23): 13203-13207.
12 Sellheyer K, Spitznas M. The fine structure of the developing human choriocapillaris during the first trimester. Graefe Arch Clin Exp Ophthalmol 1988; 226: 65-74.

13 Mann IC. The Development of the Human Eye. University Press: Cambridge, 1928.

14 Drake CJ, Fleming PA. Vasculogenesis in the day 6.5 to 9.5 mouse embryo. Blood 2000; 95: 1571-1579.

15 McLeod DS, Lutty GA. High resolution histologic analysis of the human choroidal vasculature. Invest Ophthalmol Vis Sci 1994; 35: 3799-3811.

16 Herman IM. Actin isoforms. Curr Opin Cell Biol 1993; 5: $48-55$.

17 Lutty GA, Merges C, Grebe R, Prow T, McLeod DS. Canine retinal angioblasts are multipotent. Exp Eye Res 2006; 83(1): 183-193.

18 Roy S, Hirano A, Kochen JA, Zimmerman HM. The fine structure of cerebral blood vessels in chick embryo. Acta Neuropathol 1974; 30(4): 277-285.

19 Maina JN. Systematic analysis of hematopietic, vasculogenic, and angiogenic phases in the developing embryonic avian lung, Gallus gallus variant domesticus. Tissue Cell 2004; 36: 307-322.

20 Ballermann BJ. Glomerular endothelial cell differentiation. Kidney Int 2005; 67: 1668-1671.

21 Hendrickson AE, Yuodelis C. The morphological development of the human fovea. Ophthalmology 1984; 91: 603-612. 